Table I Crude and adjusted odds ratios (ORs) and $95 \%$ confidence intervals ( $95 \% \mathrm{Cls}$ ) for developing schizophrenia according to maternal-paternal age difference

\begin{tabular}{lcccc}
\hline Parental age difference (years) & $\begin{array}{c}\text { Number in } \\
\text { cohort (\%) }\end{array}$ & $\begin{array}{c}\text { Number with } \\
\text { schizophrenia }(\%)\end{array}$ & $\begin{array}{c}\text { Crude OR } \\
(95 \% \mathrm{Cl})\end{array}$ & $\begin{array}{c}\text { Adjusted OR } \\
(95 \% \mathrm{Cl})^{\prime}\end{array}$ \\
\hline $0-\mathrm{I}^{2}$ & $10757(23)$ & $64(0.59)$ & 1.0 & 1.0 \\
$2-3$ & $12711(27)$ & $85(0.67)$ & $1.1(0.8-1.6)$ & $1.1(0.8-1.5)$ \\
$4-5$ & $9345(20)$ & $69(0.74)$ & $1.2(0.9-1.7)$ & $1.2(0.8-1.7)$ \\
$6-9$ & $9260(20)$ & $77(0.83)$ & $1.4(1.0-2.0)$ & $1.2(0.9-1.8)$ \\
$10-47$ & $4332(10)$ & $40(0.92)$ & $1.6(1.0-2.3)$ & $1.2(0.8-1.9)$ \\
Linear trend across categories & & & $1.12(1.03-1.21)$ & $1.06(0.96-1.16)^{3}$ \\
\hline
\end{tabular}

I. Adjusted for paternal age.

2. Baseline comparison group.

3. $P=0.249$.

the difference between maternal and paternal ages must also increase given the biological age threshold for motherhood. However, in younger fathers with older mothers, even large differences in parental ages is not associated with increasing risk of schizophrenia. In contrast, the association between advancing paternal age and risk of developing schizophrenia is not altered by adjusting for parental differences. The hypothesis of increasing germ cell mutations remains the most likely explanation for this association between advancing paternal age and risk of schizophrenia.

S. Zammit Department of Psychological Medicine, University of Wales College of Medicine, Heath Park, Cardiff CFI4 4XN, UK

P. Allebeck Department of Social Medicine, Gothenburg University, Sweden

C. Dalman Psychiatric Epidemiology, Stockholm Centre of Public Health, Sweden

I. Lundberg, T. Hemmingson Department of Public Health Sciences, Karolinska Institute, Stockholm, Sweden

M. J. Owen Department of Psychological Medicine, University of Wales College of Medicine, Cardiff, UK

G. Lewis Division of Psychiatry, University of Bristol, Bristol, UK

\section{Physical illness and schizophrenia}

I read with interest the report by McCreadie (2003), which concludes that the lifestyle of people with schizophrenia must give cause for concern in relation to coronary heart disease. Despite being at an increased risk of developing various physical health problems, the detection rate and treatment of physical illness among people with mental illness is very poor (Koran et al, 1989). The reasons why this vulnerable group of patients do not receive the physical health care they deserve are manifold and need to be addressed. They range from physical symptoms being misinterpreted as part of psychiatric illness by professionals, to poor social skills, lack of motivation, cognitive impairment and social isolation occurring as part of mental illness making individuals with schizophrenia less likely to report symptoms and adhere to treatment. When they do present themselves, their lack of social skills and the stigma of mental illness may also make it less likely that they receive good care (Phelan et al, 2001).

Services focusing on lifestyle changes geared to the particular needs of people with severe mental illness should be planned. Periodic medical reviews by general practitioners using essential checklists should be mandatory. Inability to clearly appreciate or describe a medical problem, compounded by a reluctance to discuss attention to medical problems in patients with schizophrenia. Thorough, routine physical examination whenever a patient is seen is the best way forward but it is doubtful whether psychiatric services have the resources and time to implement this. It is necessary for a medical orientation on the part of psychiatrists while evaluating all patients. Refresher training should be regularly provided for psychiatrists and key members of multidisciplinary community psychiatric teams. This could encompass elements of detection, management and preventive counselling (Lambert et al, 2003). To ensure appropriate care for comorbid medical problems there should be active efforts on the part of general practitioners as well as mental health teams. such problems, contributes to the lack of
Koran, L. M., Sox, H. C. Jr, Marton, K. I., et al (1989) Medical evaluation of psychiatric patients. I. Results in a state mental health system. Archives of General Psychiatry, 46, 733-740.

Lambert, T. J. R., Velakoulis, D. \& Pantelis, C. (2003) Medical comorbidity in schizophrenia. Medical journal of Australia, 178 (suppl. 5), S67-S70.

McCreadie, R. G. (2003) Diet, smoking and cardiovascular risk in people with schizophrenia. Descriptive study. British Journal of Psychiatry, $\mathbf{1 8 3}$, 534-539.

Phelan, M., Stradins, L. \& Morrison, S. (200I)

Physical health of people with severe mental illness. BMy, 322, 443-444.

C.T. Sudhir Kumar The Maudsley Hospital,

Denmark Hill, London SE5 8AZ, UK

\section{Antidepressant effects of repetitive transcranial magnetic stimulation}

The report by Martin et al (2003) seems in conflict with previous meta-analyses of repetitive transcranial magnetic stimulation (rTMS) (Holtzheimer et al, 2001; McNamara et al, 2001; Burt et al, 2002). We wish to provide a broader context for interpreting these results.

The analysis by Martin et al was designed to minimise type 1 error - to identify the level of confidence that can be placed in purported antidepressant effects of rTMS. It combined only studies with similar methodologies, included only studies that met high standards of randomisation and blinding, and analysed only end-point depression ratings (rather than analysing change scores or controlling for baseline depression severity). With this approach, the review found a statistically significant effect size for high-frequency $(>1 \mathrm{~Hz})$ rTMS applied to the left prefrontal cortex $(-0.35,95 \%$ CI -0.66 to $-0.04, P=0.03)$, but did not find evidence that antidepressant effects were clinically significant or that they persisted over time.

The other meta-analyses attempted to minimise type 2 error - to identify whether there is reason to believe that rTMS might have significant antidepressant properties warranting further investigation. They combined studies with different methodologies and calculated effect sizes based on changes in depression severity over time. Such a technique can be important when analysing studies where different treatment arms may start at different baselines. Using these analytic techniques, prior metaanalyses found effect sizes for highfrequency, left prefrontal rTMS ranging from 0.5 to 0.8 , suggesting that rTMS does 